\title{
Twisting the bone marrow stem cell niche
}

\author{
Haixia Niu $^{1}$ and Jose A. Cancelas ${ }^{1,2}$ \\ ${ }^{1}$ Division of Experimental Hematology and Cancer Biology, Cancer \& Blood Diseases Institute, Cincinnati Children's Hospital Medical Center, \\ 3333 Burnet Ave., Cincinnati, and ${ }^{2}$ Hoxworth Blood Center, University of Cincinnati Academic Health Center, OH, USA
}

E-mail: jose.cancelas@uc.edu

doi:10.3324/haematol.2018.206029

$\mathrm{H}$ ematopoietic stem cells (HSC) are the source of blood cells over the whole lifespan of mammals. HSC locate in the bone marrow microenvironment in the so-called bone marrow stem cell niche. This niche consists of multiple cell types, such as endothelial cells, mesenchymal stem cells (MSC), CXCL12-expressing adventitial cells (CAR cells), nonmyelinating Schwann cells, pericytes, osteoblasts, adipocytes and hematopoietic cells including osteoclasts, regulatory $\mathrm{T}$ cells, macrophages and neutrophils. ${ }^{1}$ Our understanding of the specific contributions of each of these populations derives from the use of constitutional or inducible genetic murine models in which more-or-less specific niche cell populations are targeted for deletion, or for the introduction of loss-of-function or gain-of-function mutations. However, the complicated but precise crosstalk between HSC and specific bone marrow HSC niche cells is still poorly understood. In this issue of the journal, Liu et al. report on their discovery that inducible deficiency of Twist1 in the bone marrow HSC niche results in a broad alteration of multiple niche cell types as well as a reduced production of major HSC supportive factors. The dysregulated Twist1-deficient mesenchymal bone marrow HSC niche reduces HSC homing and retention, impairs HSC selfrenewal and induces myeloid-biased differentiation. Furthermore, Twist1 deficiency in the bone marrow HSC niche accelerates the progression of MLL-AF9-induced acute myeloid leukemia (AML), partially through upregulated Jagged2/Notch signaling. ${ }^{2}$

Three significant aspects of this study need to be highlighted. First of all, the discovery adds new mechanisms to our understanding of how the bone marrow HSC niche regulates HSC function. Secondly, the findings provide cumulative evidence that remodeling of the bone marrow HSC niche plays an important role in leukemogenesis and is, therefore, a potential drug target for therapy. Last but not least, the authors identified a new function of the transcription factor Twist1 which participates in normal and malignant hematopoiesis.

\section{Niche Twist1 deficiency impairs hematopoietic stem cell function, homing and retention}

Twist proteins are members of the basic Helix-Loop-Helix transcription factor family with highly conserved sequences. There are two Twist proteins, Twist1 and Twist2. They are well-known for their essential functions in development. ${ }^{3}$ In the hematopoietic system, Twist1 is expressed in HSC and progenitors, while Twist 2 is expressed in more differentiated myeloid lineage cells. However, the function of Twist1 in hematopoiesis has not been well studied. Earlier studies by the same group demonstrated that Twist1 is a regulator of HSC self-renewal and lineage commitment. ${ }^{4}$ Twist1 also participates in the regulation of MSC function and in MSC selfrenewal. Twist1 overexpression in MSC increased C-X-C motif chemokine 12 ligand (Cxcl12) production, which enhanced the capacity to maintain human HSC and progeni- tors in stromal-dependent long-term culture-initiating cell assays. Twist1-deficient heterozygous mice showed reduced numbers of MSC in the bone marrow. ${ }^{5}$ Twist1 also regulates the cell fate and commitment of MSC. Twist1 silencing enhanced in vitro and in vivo osteogenic differentiation of human adipose-derived MSC by triggering the activation of BMP-ERK-FGF signaling and TAZ upregulation. Silencing Twist1 in a murine MSC cell line, C3H10T1/2, enhanced osteogenic differentiation. ${ }^{6}$ Using a chimeric mouse model, Liu et al., found that Twist1 deletion in the bone marrow niche leads to decreased numbers of MSC and mature osteoblasts, ${ }^{2}$ findings which are consistent with already reported data. However, both sinusoidal and arteriolar endothelial cell numbers increased, consistent with an increase in the number of microvessels. This finding contrasts with that of earlier studies in which Twist1 upregulation, as identified in numerous carcinomas, promoted neo-angiogenesis. ${ }^{7}$ These distinct roles of Twist1 in tumor epithelial cells may be attributed to the different microenvironment found in solid tumors compared with the bone marrow HSC niche. More studies need to be performed to address this difference.

Most HSC are retained in the BM niche in a quiescent state by interacting with the niche cells. The inducible deficiency of Twist1 in the bone marrow HSC niche promoted cell cycle entry of long-term HSC, impaired long-term HSC self-renewal and biased HSC differentiation to the myeloid lineage. The CXCL12-CXCR4 axis is essential for HSC retention in the bone marrow. ${ }^{8}$ Granulocyte-colony stimulating factor (G-CSF), an endogenously expressed cytokine secreted by MSC, osteolineage cells, endothelial cells and macrophages, induces mobilization of quiescent HSC through downregulation of bone marrow expression of CXCL12.,10 Liu et al. found that the inducible deficiency of Twist1 impaired HSC homing and promoted HSC mobilization, effects associated with decreased expression of CXCL12 and other key cytokines/chemokines crucial for bone marrow HSC retention, such as VCAM, SCF, and Angpt1 in mutant bone marrow MSC, and increased GCSF expression in bone marrow osteolineage cells and macrophages. ${ }^{2}$ Together with their earlier finding, ${ }^{4}$ these data indicate that Twist1 regulates HSC function in both a cellautonomous and non-cell-autonomous manner.

An earlier study by the same group showed that Twist1 regulates HSC myeloid differentiation by activating the myeloid transcription factors PU.1 and Gata1 and downregulating the lymphoid transcription factor Gata3. ${ }^{4}$ A Twist1-deficient bone marrow HSC niche promoted early myeloid differentiation. ${ }^{2}$ However, the underlying molecular mechanism was not identified. Twist2, a homolog of Twist1, has also been identified as a major negative regulator of myeloid cell development and the pro-inflammatory responses of these cells. ${ }^{11}$ Twist2 is expressed in granulocyte-macrophage progenitors and inhibits their proliferation and differentiation into macrophages, neutrophils and basophils through direct interactions and inhibi- 


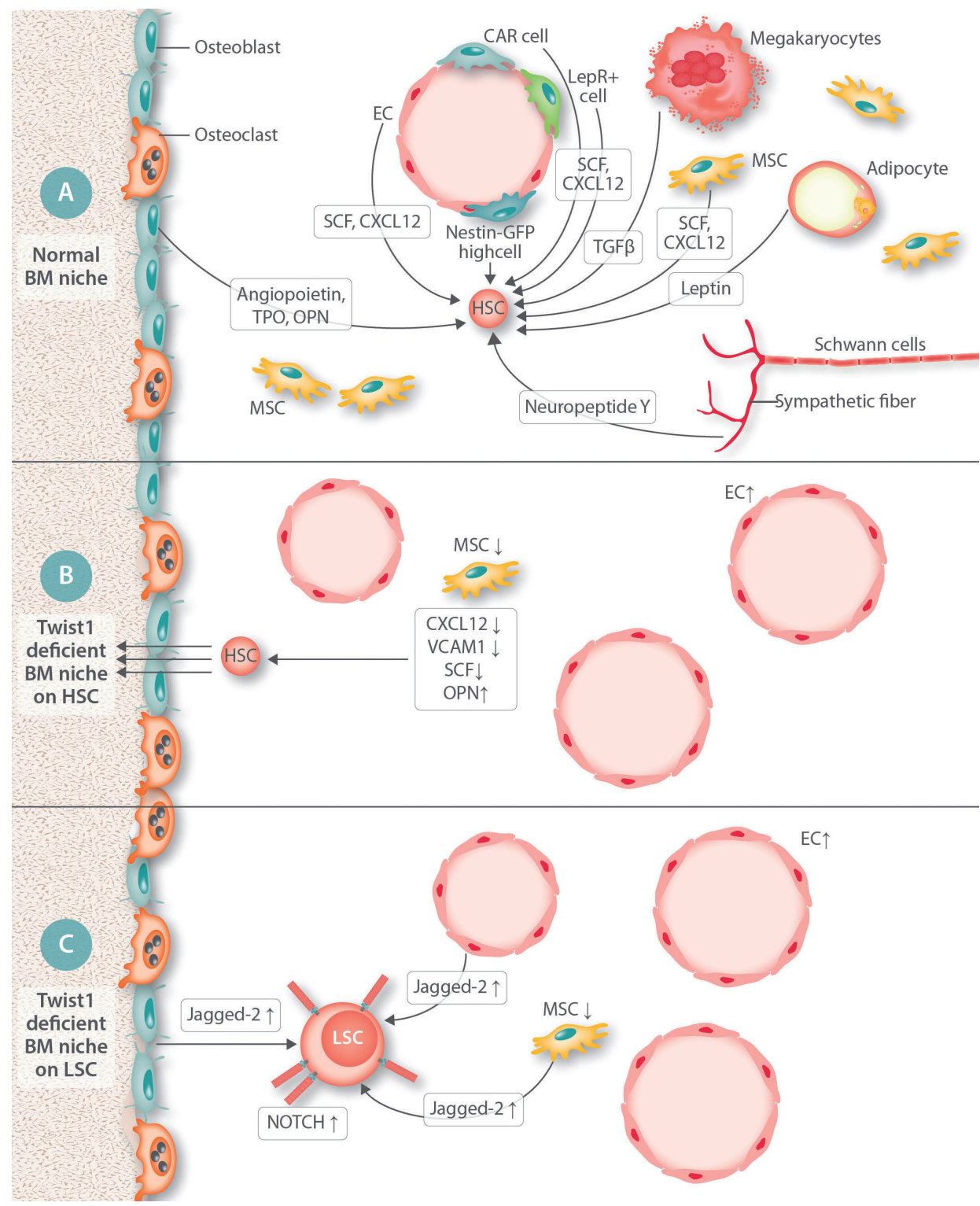

Figure 1. Effect of inducible deficiency of Twist1 in the bone marrow niche. (A) Normal bone marrow (BM) niche. The normal BM niche constists of multiple cell types, including nonhematopoietic endothelial cells (EC), CXCL12-expressing adventitial cells (CAR cells), Leptin-receptor-positive cells (LepR+ cells), Nestin - green fluorescent protein high-expressing cells [Nestin-GFP(high) cells], myelinated fibers (surrounded by Schwann cells) and non-myelinated sympathetic fibers; mesenchymal stem cells (MSC); osteoblasts; and adipocytes; and hematopoietic cells that include regulatory T cells (Treg), macrophages (Mac) and osteoclasts (OC). The mechanisms used by these cells to control HSC activity include expression of short-acting hormones, cytokines and chemokines, leptin, stem cell factor (SCF); angiopoietin; thrombopoietin (TPO); C-X-C motif chemokine 12 (CXCL12); transforming growth factor-beta (TGF $\beta$ ); neurotransmitters (norepinephrine and neuropeptide $Y$ ) and purine nucleotides (ATP and adenosine); extracellular proteins [osteopontin (OPN), fibronectin, and others] and direct cell-to-cell, contact-dependent interactions (e.g. CXCL12-CXCR4; integrinVCAM1) and transfer of organelles and secondary messengers. (B) The effect of a Twist1-deficient BM niche on HSC. Twist1 deficiency in BM niche causes EC expansion and angiogenesis, depletion of MSC, increased OPN production and decreased intramarrow levels of CXCL12, VCAM1 and SCF production while OPN production increases, which impairs HSC retention in the BM niche and promotes their mobilization to the periphery. (C) The effect of a Twist1-deficient BM niche on leukemic stem cells (LSC). The Twist1-deficient BM niche promotes acute myelogenous leukemia (AML) after transformation by the MLL-AF9 oncogene. The mechanism involves upregulated Notch signaling through upregulation of the production of local Jagged-2 (expressed by $\mathrm{EC}$, osteoblasts and MSC) and the membrane expression of Notch receptors on LSC.

tion of Runx1 and C/EBP $\alpha$. In mature myeloid cells, Twist2 negatively regulates pro-inflammatory responses by inhibiting the expression of pro-inflammatory cytokines such as interleukin-12, interferon- $\gamma$, interleukin-1, tumor necrosis factor- $\alpha$, interleukin- 6 , and macrophage inflammatory protein- $1 \alpha$, through the inactivation of $\mathrm{c} / \mathrm{EBP} \alpha$ and NF-kB. According to these data, Twist1 and Twist2 play opposite roles in myeloid differentiation although how the opposing functions of these two homologs in myeloid differentiation are balanced remains unclear.

\section{Twist1 in acute myeloid leukemia}

Twist1 plays an important role in the process of epithelial-mesenchymal transition. The overexpression of Twist1 has been described as a poor prognostic factor in numerous epithelial-derived malignancies such as breast cancer, prostate cancer, colorectal cancer, bladder cancer, melanoma, hepatocellular carcinoma and neck carcinoma. ${ }^{7}$ Twist 1 has been found to be overexpressed in mononuclear cells from the bone marrow of patients with AML and chronic myeloid leukemia, ${ }^{12}$ with a strong correlation between the expression of Twist1 and Bmi-1, an essential polycomb complex group with a fundamental role in the maintenance of leukemia stem cells. In fact, AML patients whose blasts overexpress Twist1 have a more aggressive clinical phenotype, with a good response to the cell cycle phase-specific agent cytarabine but not to the non-cell cycle phase-specific anthracycline, daunorubicin. ${ }^{13}$ It also been shown that Twist1 expression is augmented in the HSC and progenitor compartment and decreased in bone marrow stromal cells from patients with myelodysplastic syndrome. ${ }^{14}$ However, whether Twist1 in the bone marrow niche participates in AML pathogenesis is not clear.

MLL-AF9 is an oncoprotein, a product of chromosome 
translocation $\mathrm{t}(9 ; 11)(\mathrm{p} 22 ; \mathrm{q} 23)$, typically associated with the M4 or M5 French-American-British subtypes of human AML. An MLL-AF9-induced AML mouse model is widely used to study AML. Hanoun et al. showed that MLL-AF9induced AML cells disrupt bone marrow HSC niche function through alteration of the niche compartments and decrease of the expression of MSC-derived Cxcl12, Scf, Vcam1, and increased expression of Opn. ${ }^{15}$ These phenotypes in the MLL-AF9-induced AML bone marrow niche mirrored those in the Twist1-deficient bone marrow niche observed by Liu et al. ${ }^{2}$ They also found that a Twist1-deficient niche promoted progression of MLL-AF9-induced AML. By using RNAsequencing, the authors found that Jagged-2 is significantly increased in the stromal cells from the Twist1-deficient bone marrow niche and that Notch receptors are upregulated on leukemia cells. Pharmaceutical inhibition of Notch signaling partially inhibited the leukemia progression. These data indicate that AML progression is a closed loop, AML cells impair the normal bone marrow niche and alter the niche to support AML cell survival and progression. Targeting the leukemia cell - bone marrow niche loop could be an efficient strategy for leukemia therapy. Jagged-2/Notch signaling is a potential target.

\section{Relevance of understanding the hematopoietic niche in human disease}

Bone marrow failure syndromes, including aplastic anemia and myelodysplastic syndromes result from cell- and non-cell-autonomous dysregulation. Similarly, there is increasing evidence of the role of the bone marrow niche in leukemogenesis. Non-cell-autonomous dysregulation is linked to changes in the signals from innate and adaptive immune environments, and endothelial and mesenchymal lineage cells. Big data analyses based on matrix analyses of combined large data sets from single-cell RNA sequencing, flow/mass cytometry, metabolomics and proteomics mass spectrometry, as well as sophisticated microanatomical analyses are generating multiple hypotheses on the specific dissection of the interacting signal networks that connect the HSC niche cells of the bone marrow. These hypotheses require exquisite analysis and validation in mutant animal models and in therapeutic approaches when pharmacological tools to specific targets allow analyses of efficacy and safety in patients with hematologic diseases.

\section{References}

1. Gao X, Xu C, Asada N, Frenette PS. The hematopoietic stem cell niche: from embryo to adult. Development. 2018;145(2). pii: dev139691.

2. Liu X, Ma Y, Li R, et al. Niche TWIST1 is critical for maintaining normal hematopoiesis and impeding leukemia progression. Haematologica 2018;103(12):1969-1979.

3. Barnes RM, Firulli AB. A twist of insight - the role of Twist-family bHLH factors in development. Int J Dev Biol. 2009;53(7):909-924.

4. Dong CY, Liu XY, Wang N, et al. Twist-1, a novel regulator of hematopoietic stem cell self-renewal and myeloid lineage development. Stem Cells. 2014;32(12):3173-3182.

5. Arthur A, Cakouros D, Cooper L, et al. Twist-1 enhances bone marrow mesenchymal stromal cell support of hematopoiesis by modulating CXCL12 expression. Stem Cells. 2016;34(2):504-509.

6. Miraoui H, Severe N, Vaudin P, Pages JC, Marie PJ. Molecular silencing of Twist1 enhances osteogenic differentiation of murine mesenchymal stem cells: implication of FGFR2 signaling. J Cell Biochem. 2010;110(5):1147-1154

7. Zhao Z, Rahman MA, Chen ZG, Shin DM. Multiple biological functions of Twist1 in various cancers. Oncotarget. 2017;8(12):2038020393.

8. Peled A, Petit I, Kollet $O$, et al. Dependence of human stem cell engraftment and repopulation of NOD/SCID mice on CXCR4. Science. 1999;283(5403):845-848.

9. Greenbaum A, Hsu YM, Day RB, et al. CXCL12 in early mesenchymal progenitors is required for haematopoietic stem-cell maintenance. Nature. 2013;495(7440):227-230.

10. Mendez-Ferrer S, Frenette PS. Hematopoietic stem cell trafficking: regulated adhesion and attraction to bone marrow microenvironment. Ann N Y Acad Sci. 2007;1116:392-413.

11. Sharabi AB, Aldrich $M$, Sosic $D$, et al. Twist-2 controls myeloid lineage development and function. PLoS Biol. 2008;6(12):e316.

12. Wang N, Guo D, Zhao YY, et al. TWIST-1 promotes cell growth, drug resistance and progenitor clonogenic capacities in myeloid leukemia and is a novel poor prognostic factor in acute myeloid leukemia. Oncotarget. 2015;6(25):20977-20992.

13. Chen CC, You JY, Gau JP, et al. Favorable clinical outcome and unique characteristics in association with Twist1 overexpression in de novo acute myeloid leukemia. Blood Cancer J. 2015;5:e339.

14. Li X, Marcondes AM, Gooley TA, Deeg HJ. The helix-loop-helix transcription factor TWIST is dysregulated in myelodysplastic syndromes. Blood. 2010;116(13):2304-2314.

15. Hanoun M, Zhang D, Mizoguchi T, et al. Acute myelogenous leukemia-induced sympathetic neuropathy promotes malignancy in an altered hematopoietic stem cell niche. Cell Stem Cell. 2014;15(3):365375 .

\section{Assessment of iron deficiency}

\section{Chaim Hershko}

Professor Emeritus, Department of Hematology, Shaare Zedek Medical Center, Jerusalem, Israel

E-mail:hershkoc@netvision.net.il

doi:10.3324/haematol.2018.205575

A ccording to a study involving 187 countries, ${ }^{1}$ the global prevalence of anemia in 2010 was 33\% and it was responsible for 68 million years lived with disability. Iron deficiency was the top cause of anemia, with children below 5 years and women having the highest burden. In addition to iron deficiency, which was the most common etiology globally, other leading causes of anemia vary widely by geography, age, and sex.
Traditionally, the diagnosis of iron deficiency anemia (IDA) rests on simple measurements of serum iron, transferrin and ferritin in subjects with microcytic hypochromic anemia. However, iron deficiency and other conditions associated with anemia, such as the anemia of chronic disease and hemoglobinopathies often coexist, requiring further refinement of diagnostic strategies. The study reported by Kanuri et al. ${ }^{2}$ in this issue represents an effort to opti- 